\title{
Influência da privação visual no desempenho do teste de 10RM em homens treinados
}

\section{Influence of visual privacy on 10RM test performance in trained men}

\section{Influencia de la privación visual en el rendimiento de la prueba de 10RM en hombres entrenados}

\author{
iD Krystal Alonso \\ Universidade Federal do Rio de Janeiro, Rio de Janeiro, Rio de Janeiro, Brasil \\ Krika_kry@hotmail.com \\ iD) Gabriel Paz \\ Universidade Federal do Rio de Janeiro, Rio de Janeiro, Rio de Janeiro, Brasil \\ gabriel.andrade.paz@gmail.com
}

\begin{abstract}
Resumo: O objetivo do estudo foi avaliar e comparar a carga deslocada no teste de 10 RM sob as condições com e sem a privação visual. A amostra foi composta por 13 homens treinados em força (idade 27,7 \pm 3 anos). Foram realizadas quatro sessões para teste e reteste de 10 RM nos exercícios: Supino horizontal (SH) e Hack Machine (HM). Para a análise estatística, foi utilizado o test T pareado para comparar os resultados obtidos. Como resultado obtivemos que a carga deslocada com privação visual (PV) no (SH) foi de $150 \mathrm{~kg}$ e sem privação (SPV) foi de $135 \mathrm{~kg}$, e para o HM CPV a carga foi de 54 kg e SPV 48 kg. Com base nos resultados apresentados, conclui-se que a carga deslocada CPV foi significativamente maior do que com a visualização das cargas.
\end{abstract}

Palavras-chave: Treinamento de resistência. Retroalimentação sensorial. Força muscular. 
Abstract: The objective of the study was to evaluate and compare the displaced load in the $10 \mathrm{RM}$ test under conditions with and without visual deprivation. The sample consisted of 13 men trained in strength (age $27.7 \pm 3$ years). Four sessions were used to test and retest 10 RM in the exercises: Horizontal Supine (SH) and Hack Machine (HM). For the statistical analysis, the paired T-test was used to compare the results obtained. As a result we found that the displaced load with visual deprivation (SH) in the SH was $150 \mathrm{~kg}$ and without deprivation (SPV) was $135 \mathrm{~kg}$ and for the HM CPV the load was $54 \mathrm{~kg}$ and the SPV was $48 \mathrm{~kg}$. Based on the results presented, it was concluded that the CPV displaced load was significantly higher than with the visualization of the loads.

Keywords: Resistance training. Sensory feedback. Muscle strength.

Resumen: El objetivo del estudio fue evaluar y comparar la carga desplazada en la prueba de 10 RM bajo las condiciones con y sin la privación visual. La muestra fue compuesta por 13 hombres entrenados en fuerza (edad 27,7 \pm 3 años). Se realizaron cuatro sesiones para prueba y reteste de $10 \mathrm{RM}$ en los ejercicios: Supino horizontal (SH) y Hack Machine (HM). Para el análisis estadístico, se utilizó el test T pareado para comparar los resultados obtenidos. Como resultado obtuvimos que la carga desplazada con privación visual (PV) en el (SH) fue de 150 kg y sin privación (SPV) fue de $135 \mathrm{~kg}$ y para el HM CPV la carga fue de $54 \mathrm{~kg}$ y SPV $48 \mathrm{~kg}$. Con base en los resultados presentados, se concluye que la carga desplazada CPV fue significativamente mayor que con la visualización de las cargas.

Palabras clave: Entrenamiento de resistencia. Retroalimentación sensorial. Fuerza muscular.

Submetido em: 19-12-2018

Aceito em: 13-04-2020 
Influência da privação visual no desempenho do teste de 10rm em homens treinados Krystal Alonso • Gabriel Paz

\section{Introdução}

O treinamento de força (TF) pode ser compreendido como uma série de contrações musculares voluntárias impostas contra uma resistência/carga externa, esta pode ser mensurada facilmente, executada a partir de contrações concêntricas, excêntricas ou isométricas (FLECK; KRAEMER, 2017). No âmbito da ciência do esporte e do exercício, Knuttgen e Kraemer (1987) definem a força como a quantidade máxima de força que um músculo ou grupamento muscular é capaz de gerar em um padrão de movimento específico, sendo este realizado a uma determinada velocidade.

Inúmeros são os benefícios já documentados pela literatura científica decorrentes da prática do TF, e suas aplicações não se restringem somente a atletas ou a indivíduos que almejam meIhorias estéticas. A prática regular do TF desenvolve vários componentes da aptidão física, destacando-se o aumento de força máxima, potência, resistência e hipertrofia muscular (ACSM, 2009; HARRIESS; LUBANS; CALLISTER, 2015; SCHOENFELD, OGBORN, KRIEGER, 2017). Há, ainda, benefícios em outros parâmetros fisiológicos, tais como: melhoras no sistema cardiovascular, perfil lipídico, composição corporal e saúde óssea (DE MATOS et al., 2009; ECKEL et al., 2014).

O teste de uma repetição máxima (1RM) tem sido amplamente empregado para determinar a força máxima dinâmica, sendo considerado um teste de campo padrão-ouro (BAECHLE; EARLE, 2000; RHEA et al., 2003). Como forma alternativa, é possível utilizar o teste de 10 repetições máximas (10RM), que pode ser mais aplicável e mais seguro para determinadas populações ou grupamentos musculares específicos (BAECHLE; EARLE, 2000), sendo que o ajuste da intensidade para um determinado número de repetições máximas é satisfatoriamente cumprido quando este procedimento é empregado. Nesse contexto, existem algumas variáveis que podem influenciar no desempenho dos testes de força. Entre elas, 
Influência da privação visual no desempenho do teste de 10rm em homens treinados Krystal Alonso • Gabriel Paz

é possível destacar a visualização da carga que será utilizada e a visualização da execução do movimento proposto, pois o fato de não poder visualizar o que está sendo feito tira do indivíduo o controle visual sobre o espaço durante as ações ligadas à mobilidade articular (ATKINS; FISER; JACOBS, 2001). Apesar disso, é pertinente salientar que a privação visual durante a realização de exercícios resistidos recebe pouca atenção por parte dos pesquisadores e, portanto, pouco se sabe sobre a sua relevância durante a condução de testes de repetições máximas.

Nesse sentido, em estudo conduzido por Maior e colaboradores (2007), os autores analisaram a influência da privação visual sobre o desempenho no teste de 1 RM em 12 homens treinados em força. Os achados demonstraram que a carga mobilizada nos exercícios: Supino Horizontal, Leg Press $45^{\circ}$ e Puxada pela Frente, realizados com privação visual, foi significativamente maior quando comparado ao protocolo sem privação visual, demonstrando, assim, que ao visualizar a carga imposta, o indivíduo pode subestimar a sua capacidade de mobilizar determinada carga.

Até o presente momento, não foram encontrados na literatura estudos verificando a influência da privação visual na aplicação do teste de 10RM. Como já é sabido, os praticantes de TF tendem a subestimar a sua capacidade de erguer a carga, podendo gerar resultados errôneos. O intuito do trabalho é promover uma maior confiabilidade nos testes de campo, gerando resultados mais fidedignos. Portanto, o objetivo do estudo foi comparar os valores obtidos no teste de 10 RM nos exercícios: Supino vertical (SV) e Hack Machine (HM), realizado por homens com experiência no TF, sob as condições com e sem privação visual.

Como hipótese básica do estudo, supõe-se que o desempenho no protocolo com privação visual seja melhor em relação ao teste sem privação visual. 
Influência da privação visual no desempenho do teste de 10rm em homens treinados Krystal Alonso - Gabriel Paz

\section{Métodos}

Trata-se de um estudo do tipo experimental crossover visando à comparação entre testes de 10 RM realizados com e sem privação visual. Foi comparada a carga mobilizada nos exercícios SV e HM.

Participaram do presente estudo 13 homens treinados em força (idade 27,7 \pm 3 anos; peso 77,4 $\pm 12 \mathrm{~kg}$; altura 1,75 $\pm 7,9 \mathrm{~cm}$; IMC 25 $\left.\pm 3,5 \mathrm{~kg} / \mathrm{m}^{2}\right)$, selecionados por conveniência a partir dos seguintes critérios de inclusão: a) Treinados em força com, no mínimo, seis meses de experiência no TF; b) Sem registro prévio de lesões de qualquer ordem nos últimos 12 meses. Como critério de exclusão, foram adotados: a) PAR-Q positivo; b) Uso de recursos ergogênicos que possam influenciar nos resultados.

Os participantes foram orientados a não ingerir substâncias do tipo estimulante (cafeína ou álcool) e não realizar qualquer atividade física no dia das avaliações e no dia anterior.

Todos os participantes responderam ao Questionário de Prontidão para Atividade Física (PAR-Q) e assinaram o termo de consentimento livre e esclarecido antes da participação no estudo. Este foi elaborado de acordo com a Declaração de Helsinki e resolução 466/12 do Conselho Nacional de Saúde (2012).

\section{Determinação da carga de 10 repetições máximas}

As avaliações ocorreram em quatro visitas, conforme ilustrado na figura 1. De forma randomizada, foram utilizadas duas sessões para teste e reteste das 10 RM sem a privação visual e outras duas 
Influência da privação visual no desempenho do teste de 10rm em homens treinados Krystal Alonso - Gabriel Paz

sessões para o protocolo com a privação visual. No primeiro encontro foi feita a coleta dos dados antropométricos.

A carga utilizada no teste de 10 RM para os exercícios SV e HM foi determinada em dois dias distintos com intervalo de 48-72 horas entre eles, sendo utilizada entrada alternada para ambos os exercícios. Os procedimentos do teste foram seguidos de acordo com o protocolo proposto por Baechle e Earle (2000) e a carga inicial foi selecionada a partir do peso habitualmente utilizado nos treinamentos pelo participante. O teste era interrompido no momento em que o avaliado perdesse a técnica adequada para a execução do movimento ou atingisse a falha muscular momentânea concêntrica em 10 RM.

Para reduzir a margem de erro no teste, instruções padronizadas foram devidamente explanadas previamente, de forma que o avaliado estivesse a par de toda a rotina que envolve a coleta de dados. $\mathrm{O}$ avaliado foi instruído sobre a técnica de execução do exercício e o avaliador permaneceu atento à posição adotada pelo praticante durante o teste, considerando que pequenas variações no posicionamento das articulações envolvidas no movimento poderiam acionar outros grupamentos musculares, podendo levar a interpretações errôneas dos escores obtidos. Estímulos verbais foram realizados com o intuito de manter a motivação elevada. Os intervalos entre uma tentativa e outra em cada exercício durante o teste de 10RM foram fixados entre três e cinco minutos. Após a obtenção da carga em um determinado exercício, intervalos acima de 10 minutos foram dados, antes de se realizar o próximo exercício.

Os aparelhos contaram com limitadores de amplitude para que todos apresentassem a mesma amplitude de movimento e o teste fosse padronizado também nesse sentido; isso foi feito tanto no teste sem, como no com privação visual. As cargas adicionais utilizadas no estudo foram previamente aferidas em balança de precisão. Dessa forma, para impedir a visualização da carga empregada no protocolo com privação visual, óculos de natação foram vedados para esta finalidade. 
Influência da privação visual no desempenho do teste de 10rm em homens treinados

Figura 1. Desenho experimental do estudo.

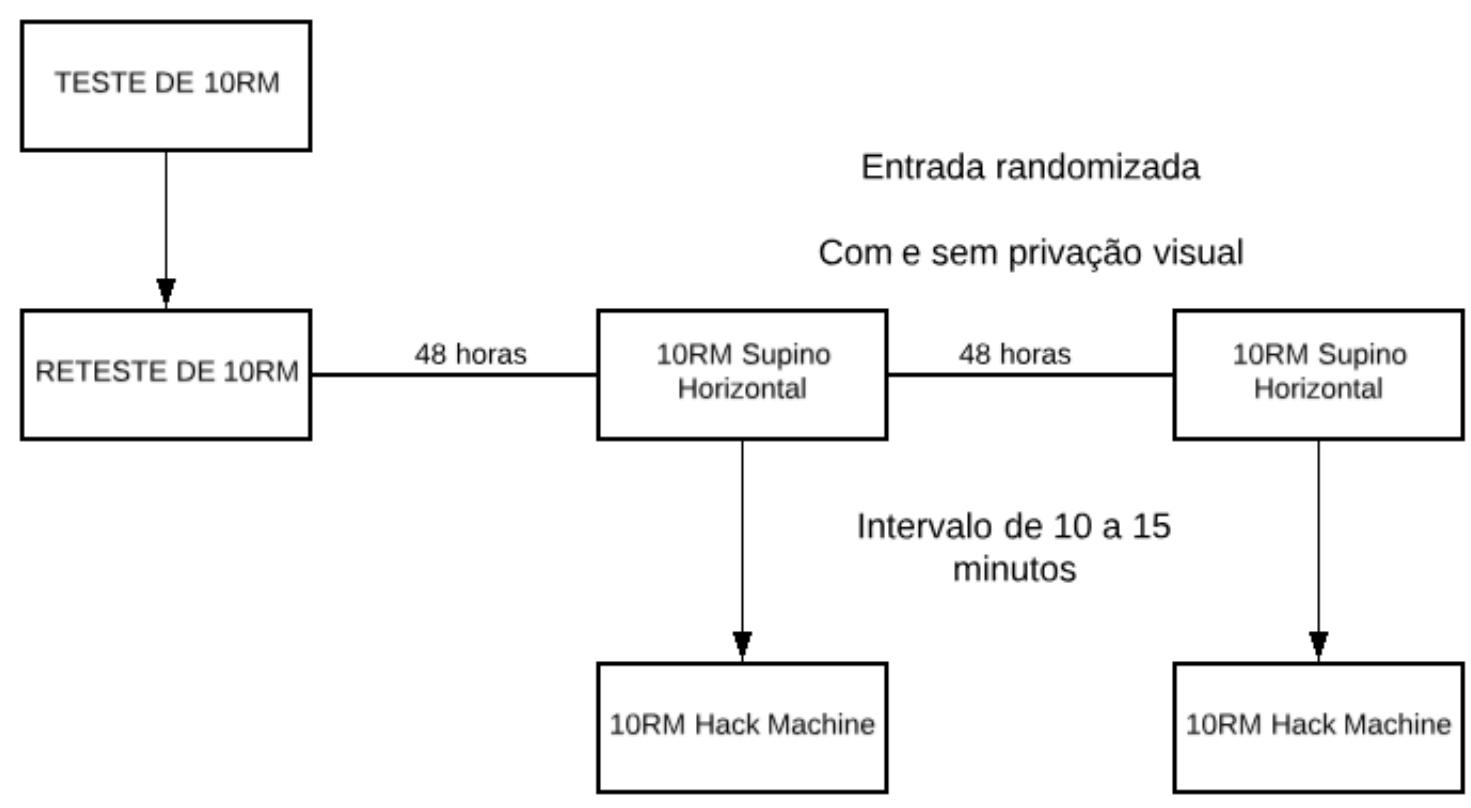

\section{Instrumentos}

Os equipamentos utilizados foram: balança com estadiômetro profissional digital Balmak New Bk-300 Fan, aparelho de musculação Supino vertical aberto wellnessby impulse e HackMachine marca Righetto FS3030 e óculos de natação marca Speedo Freestyle 3.0 vedado para o protocolo com privação visual.

\section{Tratamento estatístico}

Para a análise estatística, foi utilizado o test T student pareado para comparar os resultados dos testes de 10RM com e sem privação visual. Em todos os casos foi adotado um nível de significância de $p<0,05$. As análises foram realizadas pelo softwareIBM SPSS Statistic 22.0 e a confecção dos gráficos foi realizada por meio do software GraphPadPrism 5.0. 
Influência da privação visual no desempenho do teste de 10rm em homens treinados Krystal Alonso • Gabriel Paz

\section{Resultados}

A caracterização da amostra está expressa em média e desvio padrão na tabela 1.

Tabela 1. Característica da amostra.

\begin{tabular}{ccccc}
\hline \multicolumn{5}{c}{ CARACTERIZAÇÃO DA AMOSTRA } \\
SITUAÇÃO & IDADE & PESO & ALTURA & IMC \\
MÉDIAS & 27,7 & 77,4 & 175,3 & 25,3 \\
DESVIO PADRÃO & $\pm 3,0$ & $\pm 12,0$ & $\pm 7,9$ & $\pm 3,5$ \\
\hline
\end{tabular}

Os resultados obtidos no presente estudo, comparando os métodos com e sem privação visual no teste de 10 RM, mostraram aumentos significativos em maior mobilização de carga quando os indivíduos utilizaram o protocolo com privação visual em comparação ao protocolo sem privação visual no exercício $\mathrm{SH}(\mathrm{p}=$ 0,0001). Os dados podem ser observados no gráfico 1.

Gráfico 1. Carga obtida no teste de 10RM no supino horizontal.

\section{Teste de 10 RM no Supino Horizontal}

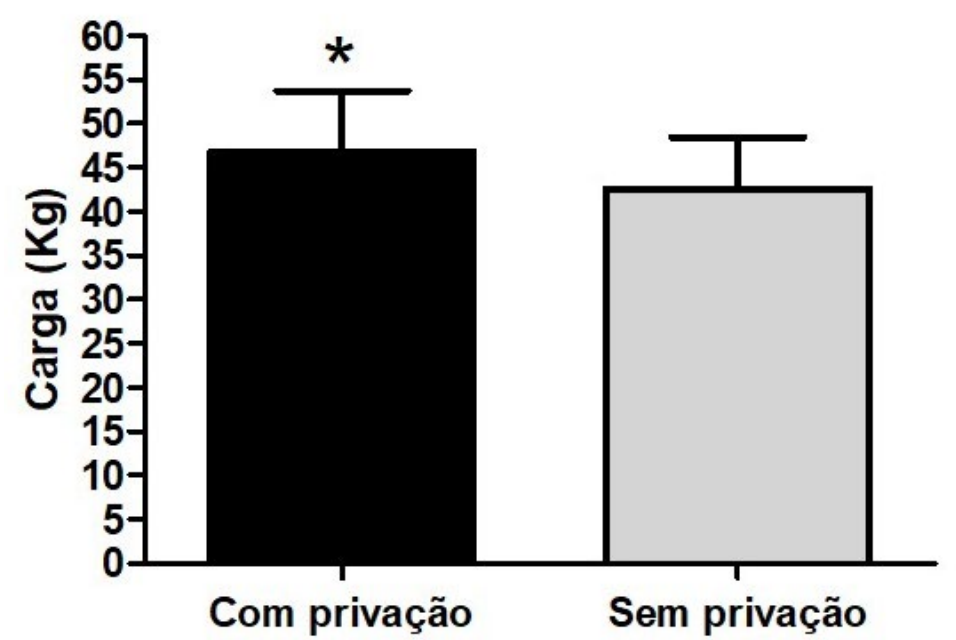


Influência da privação visual no desempenho do teste de 10rm em homens treinados Krystal Alonso - Gabriel Paz

No teste de 10 RM para o exercício HM, foi observada uma diferença estatística significativa entre os protocolos com e sem privação visual ( $p=0,0001)$, sendo que a utilização da privação visual possibilitou que os avaliados pudessem mobilizar uma carga maior. Os resultados estão inseridos no gráfico 2.

Gráfico 2. Carga obtida no teste de 10RM no hack machine.

\section{Teste de 10 RM no Hack Machine}

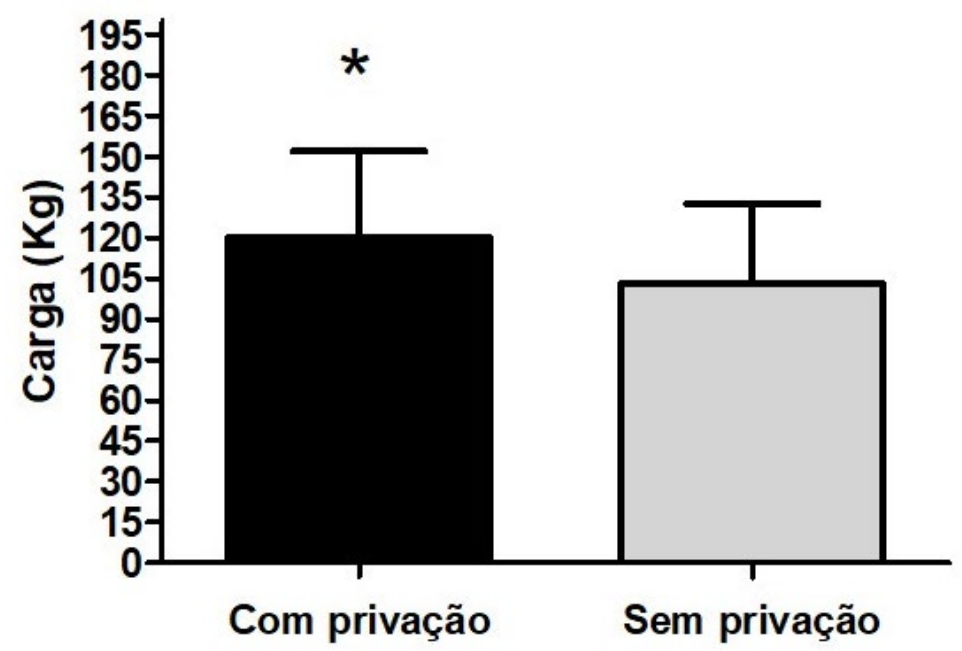

\section{Discussão}

Considerando os resultados observados, o principal achado do presente estudo demonstra que a utilização da privação visual durante a realização do teste de 10RM possibilitou um aumento significativo na mobilização de carga em relação ao protocolo sem a privação visual.

De acordo com Philbeck et al. (2001), em deslocamentos curtos, a privação visual não se mostra como um fator negativo em relação à manutenção postural porque a privação visual não exclui formas de atualização da posição e da direção realizada, mas sim de um conhecimento já adquirido da postura desejada. Essa afir- 
Influência da privação visual no desempenho do teste de 10rm em homens treinados Krystal Alonso • Gabriel Paz

mação ratifica o que foi observado no presente estudo, em que a privação visual não demonstrou nenhuma influência negativa em relação aos ajustes posturais e de equilíbrio corporal, sendo que não foram observadas mudanças no posicionamento das articulações envolvidas no movimento dos exercícios do SV e HM.

Corroborando os achados do presente estudo, Maior e colaboradores (2007) compararam o valor da carga deslocada durante a execução do teste de 1 RM com e sem privação visual. Foram avaliados 12 homens treinados em força nos exercícios Supino Horizontal (SH), Leg Press $45^{\circ}$ (LP) e Puxada pela Frente (PF). Foi observado um aumento significativo no deslocamento de carga para os testes de 1 RM com privação visual em comparação ao teste sem privação visual nos exercícios SH, LP e PF de $(5,37 \%)(p<0,0001)$, $(8,25 \%)(p<0,0001)$ e $(5,12 \%)(p<0,01)$, respectivamente. Os autores puderam concluir que com a privação visual, os indivíduos obtiveram um melhor desempenho e força ao deslocar a carga, evitando que os avaliados subestimassem suas capacidades.

Nesse contexto, para analisar se tal evento também poderia ocorrer em mulheres, Maior e Colaboradores (2010) compararam o desempenho no teste de 1RM de 11 mulheres treinadas em força nos exercícios SH, LP e PF. Os autores reportaram um aumento significativo na carga mobilizada durante o protocolo com privação visual, verificando a força máxima absoluta, sendo de $14,2 \%$ $(p<0,0001)$ no $S H, 5,6 \%(p<0,0001)$ no LP e de $10,2 \%(p<0,0001)$ na PF. Para a força máxima relativa, foram observadas diferenças significativas nos exercícios SH (11\%; $p=0,002)$, LP $(8,69 \%, p=0,000)$ e PF $(5,8 \%, p=0,000)$. Com base nos resultados apresentados, especula-se que ambos os gêneros tendem a subestimar o seu desempenho ao visualizarem a carga empregada no teste de $1 \mathrm{RM}$.

Em contrapartida, Alves et al. (2016) não encontraram diferenças significativas entre os protocolos com e sem privação visual, analisando exercícios multi e monoarticulares. Participaram do estudo 12 homens treinados em força, em que os autores compararam os valores do teste de $1 \mathrm{RM}$ em exercício multiarticular (agachamento na barra guiada [AG]) e monoarticular (rosca bíceps di- 
Influência da privação visual no desempenho do teste de 10rm em homens treinados Krystal Alonso • Gabriel Paz

reta [RD]). No exercício AG, os autores reportaram que não foram encontradas diferenças estatísticas significativas entre os protocolos para a força máxima absoluta $(p=0,422)$ e para a força relativa $(p=0,397)$. Similarmente, para o exercício RD, nenhum valor significativo foi observado tanto para a força máxima absoluta $(p=0,220)$ quanto para a força relativa $(p=0,230)$. Os autores salientam que $o$ elevado número de sessões de familiarização (4) pode ter influenciado nos escores obtidos, sendo esta uma estratégia utilizada para minimizar os efeitos da aprendizagem motora no decorrer dos testes. Em conclusão, os autores declaram que homens jovens e treinados em força não subestimam o seu desempenho e não são influenciados pelo método de privação visual, contrapondo os achados do presente estudo e de trabalhos prévios.

Como os mecanismos ainda são desconhecidos, não se sabe ao certo como o sistema proprioceptivo se comporta durante a execução de exercícios resistidos com privação visual. Nesse sentido, diferentemente do presente estudo e dos trabalhos realizados por Maior e Colaboradores (2007) e Maior e Colaboradores (2010), Alves et al. (2016) optaram pela utilização de exercícios que proporcionassem uma menor superfície de contanto entre o participante e o equipamento, por exemplo: durante o exercício HM, utilizado no presente estudo, a região posterior do tronco esteve totalmente amparada pelo equipamento, já no exercício AG, manipulado por Alves et al. (2016), não existe uma base de suporte que ofereça uma maior estabilidade para a execução do movimento. Portanto, tal fator pode ter sido crucial para o surgimento de resultados controversos. De qualquer forma, novas pesquisas devem ser realizadas para elucidar melhor tais indagações.

Maior et al. (2010) sugerem que o uso da venda nos olhos proporciona uma situação denominada de auto-eficácia, pelo fato de altas cargas possibilitarem uma tendência do indivíduo a superestimar a sobrecarga utilizada. Assim, a auto-eficácia relaciona-se ao controle de suas ações e a capacidade de ter um comportamento específico desejado (BANDURA, 1997; GEORGE; FELTZ, 1995). Além desse fator psicológico, especula-se que possa existir uma consi- 
Influência da privação visual no desempenho do teste de 10rm em homens treinados Krystal Alonso • Gabriel Paz

derável influência do sistema proprioceptivo para explicar essa melhora no desempenho do teste de 10 RM realizado no presente estudo.

O termo propriocepção refere-se ao uso do impulso sensorial enviado por vias aferentes ao sistema nervoso central para diferenciar espacialmente a posição do corpo e dos seus segmentos, bem como os movimentos e a posição articular, incluindo direção, amplitude, velocidade e tensão relativa dos tendões (BERTOTI; HOUGLUM, 2014). Considerando que existe certa dominância do sistema visual em relação ao vestibular e proprioceptivo, quando o sistema nervoso central recebe a aferência normal de todos eles (SHUMWAY-COOK, A WOOLLACOTT, 2001), em uma situação onde os olhos estão vendados ou fechados, o equilíbrio corporal passa a ser regulado pelos sistemas vestibular e proprioceptivo (LATASH et al., 2003). A partir disso, infere-se que essa melhora na resposta proprioceptiva induzida pela privação visual possa aumentar a capacidade de produção de força. Sendo assim, a influência desses mecanismos deve ser considerada na elaboração de novas pesquisas envolvendo a privação visual e o treinamento de força.

Sabe-se que a privação visual vem sendo utilizada de forma empírica há algum tempo no âmbito do treinamento de força. No entanto, para saber se a privação visual também pode ser utilizada como um método de treinamento para potencializar os resultados, estudos com intervenções em longo prazo devem ser desenvolvidos e, tendo esse conhecimento, novas estratégias com o devido respaldo científico poderão ser traçadas no que concerne à elaboração de protocolos de treinamento e confiabilidade dos testes de repetições máximas.

\section{Conclusão}

Com base nos resultados apresentados no presente estudo, conclui-se que a carga deslocada no teste de 10 RM foi significativamente maior com o uso da privação visual em comparação ao 
Influência da privação visual no desempenho do teste de 10rm em homens treinados Krystal Alonso - Gabriel Paz

teste com a visualização das cargas. Tais resultados demonstram que os indivíduos provavelmente tendem a subestimar suas capacidades físicas no teste de 10 RM ao ver a carga empregada. Em termos práticos, portanto, o recurso da privação visual deveria ser considerado na aplicação de testes de 10 RM, proporcionando, assim, resultados mais fidedignos na prescrição da intensidade nos exercícios realizados. Contudo, os mecanismos envolvidos nessa condição ainda não estão esclarecidos, fazendo-se necessária a realização de estudos futuros que possam abordar essa questão.

\section{Referências}

ACSM. Progression Models in Resistance Training for Healthy Adults. Medicine and science in sports and exercise, v. 34, n. 2 , p. 364-380, 2009.

ALVES, J. C. C. et al. Teste de uma repetição máxima em exercício multi e monoarticulares em distintos protocolos de privação visual. Revista Andaluza de Medicina del Deporte, n. xx, p. 5-9, 2016.

ATKINS, J. E.; FISER, J.; JACOBS, R. A. Experience-dependent visual cue integration based on consistencies between visual and haptic percepts. Vision Research, v. 41, n. 4, p. 449-461, 2001.

$B A E C H L E, T$. R.; EARLE, R. W. Essentials of strength training and conditioning. Champaign: Human Kinetics, 2000.

BANDURA, A. Self- Efficacy: The exercise of control. New York: Freeman, 1997.

BERTOTI, D.; HOUGLUM, P. Cinesiologia Clinica de Brunnstrom. Barueri: Manole, 2014.

DE MATOS, O. et al. Effect of specific exercise training on bone mineral density in women with postmenopausal osteopenia 
Influência da privação visual no desempenho do teste de 10rm em homens treinados Krystal Alonso - Gabriel Paz

or osteoporosis. Gynecological Endocrinology, v. 25, n. 9, p. 616-620, 2009.

ECKEL, R. H. et al. 2013 AHA/ACC guideline on lifestyle management to reduce cardiovascular risk: a report of the American College of Cardiology/American Heart Association Task Force on Practice Guidelines. Journal of the American College of Cardiology, v. 63, n. 25 Part B, p. 2960-2984, 2014.

FLECK, S.J.; KRAEMER, W. J. Fundamentos do treinamento de força muscular. Porto Alegre: Artmed Editora, 2017.

GEORGE, T. R.; FELTZ, D. L. Motivation in sport from a collective efficacy perspective. International Journal of Sport Psychology, v. 26, n. 1, p. 98-116, 1995.

HARRIES, S. K.; LUBANS, D. R.; CALLISTER, R. Systematic review and meta-analysis of linear and undulating periodized resistance training programs on muscular strength. The Journal of Strength \& Conditioning Research, v. 29, n. 4, p. 1113-1125, 2015.

KNUTTGEN, H. G.; KRAEMER, W. J. Terminology and Measurement in Exercise Performance. Journal of Strength and Conditioning Research, v. 1, n. 1, p. 1-10, 1987.

LATASH, M. L. et al. Movement sway: changes in postural sway during voluntary shifts of the center of pressure. Experimental Brain Research, v. 150, n. 3, p. 314-324, 2003.

MAIOR, A. S. et al. Resposta da força muscular em homens com a utilização de duas metodologias para o teste de 1RM. Revista Brasileira de Cineantropometria \& Desempenho Humano, v. 9, n. 2, p. 177-182, 2007.

MAIOR, A. S. et al. Resposta da força muscular em mulheres com a utilização de duas metodologias para o teste de 1RM. Revista Brasileira de Prescrição e Fisiologia do Exercício, v. 4, n. 24, p. 587-592, 2010. 
Influência da privação visual no desempenho do teste de $10 \mathrm{rm}$ em homens treinados Krystal Alonso - Gabriel Paz

PHILBECK, J. W. et al. Active control of locomotion facilitates nonvisual navigation. Journal of experimental psychology. Human perception and performance, v. 27, n. 1, p. 141-153, 2001.

RHEA, M. R. et al. A meta-analysis to determine the dose response for strength development. Medicine and Science in Sports and Exercise, v. 35, n. 3, p. 456-464, 2003.

SCHOENFELD, B. J.; OGBORN, D.; KRIEGER, J. W. Dose-response relationship between weekly resistance training volume and increases in muscle mass: A systematic review and meta-analysis. Journal of sports sciences, v. 35, n. 11, p. 1073-1082, 2017. SHUMWAY-COOK, A.; WOOLLACOTT, M. Motor control: theory and pratical applications. Baltimore: Williams \& Wilkins, 2001.

\title{
Aprovação de comitê de ética em pesquisa
}

Pesquisa aprovada pelo Comitê de Ética da Universidade Federal do Rio de Janeiro. Título: Efeito de diferentes métodos de treinamento sobre o dano muscular, desempenho de força e parâmetros cardiovasculares.

\section{Publisher}

\begin{abstract}
Universidade Federal de Goiás. Faculdade de Educação Física e Dança. Publicação no Portal de Periódicos UFG. As ideias expressadas neste artigo são de responsabilidade de seus autores, não representando, necessariamente, a opinião dos editores ou da universidade.
\end{abstract}


Influência da privação visual no desempenho do teste de 10rm em homens treinados Krystal Alonso - Gabriel Paz 